\title{
Vigilancia Tecnológica para Estudiantes Universitarios. EI Caso de la Universidad Nacional de Colombia, Sede Manizales
}

\author{
Carlos E. Marulanda ${ }^{(1,2)}$, Albeiro Hernández ${ }^{(3)}$ y Marcelo López ${ }^{(1,2)}$ \\ (1) Universidad Nacional de Colombia, Fac. de Administración, Depto. de Administración y \\ Administración de Sistemas. Campus Palogrande. Calle 27 No. 64-60, Manizales-Colombia (e-mail: \\ cemarulandae@unal.edu.co,mlopezt@unal.edu.co) \\ (2) Universidad de Caldas, Fac. de Ingeniería, Depto. de Sistemas e Informática, Calle 65 No. 26-10, \\ Manizales-Colombia. (e-mail: carlose@ucaldas,edu.co, mlopez@ucaldas.edu.co) \\ (3) Universidad Cooperativa Colombia sede Pereira, Centro de investigaciones. La Julita, Pereira- \\ Colombia. (e-mail: albeiro.hernandez@ucc.edu.co)
}

Recibido Sep. 11, 2015; Aceptado Oct. 30, 2015; Versión final Nov. 13, 2015, Publicado Abr. 2016

\begin{abstract}
Resumen
Este artículo presenta los resultados de una investigación que tiene como objetivo establecer la percepción de la vigilancia tecnológica de los estudiantes de pregrado de la Universidad Nacional de Colombia sede Manizales. La vigilancia tecnológica entendida como la captación permanente del exterior y de la propia organización sobre ciencia y tecnología para convertirla en conocimiento, no está siendo bien aprovechada por los estudiantes. Para el estudio se utilizaron métodos de observación, análisis y síntesis y un tipo de estudio descriptivo exploratorio y correlacional. Los resultados evidencian que la universidad está haciendo esfuerzos importantes desde la adquisición de herramientas y la inversión de recursos en medios de divulgación. Sin embargo, los estudiantes no lo perciben en forma adecuada y no aplican los avances. Por ejemplo, los estudiantes no están usando en forma masiva el recurso más popular disponible hoy en día como son los teléfonos móviles para hacer una adecuada vigilancia tecnológica. Este estudio aporta a la comunidad académica para la definición de una línea base que permita futuras actuaciones para potenciar la apropiación de herramientas de vigilancia tecnológica en procesos de aprendizaje.
\end{abstract}

Palabras clave: vigilancia tecnológica, tecnologías digitales, educación universitaria

\section{Technology Surveillance for University Students. The Case of the National University of Colombia, Manizales Campus}

\begin{abstract}
This article presents the results of a research that aims to establish the perception of technological surveillance of undergraduate students at the Manizales Campus of the National University of Colombia. Technological surveillance, viewed as the permanent gathering of information about science and technology to convert it into knowledge, is not being well utilized by students. For the study, methods of observation, analysis and synthesis and a type of exploratory and correlational descriptive approach were used. The results show that the university is making significant efforts by the acquisition of technological tools and investing resources in mass media diffusion. However, students do not perceive these advances in an appropriate way and are not applying the available systems. For instance, the students are not massively using one of the most popular resources such as the mobile phones to make adequate technological surveillance. This study contributes to the academic community to define a baseline to future actions to enhance the use of technological surveillance tools in learning processes.
\end{abstract}

Keywords: Technological surveillance, digital technologies, higher education 


\section{INTRODUCCIÓN}

El mundo organizacional de hoy se caracteriza por una marcada velocidad para la toma de decisiones, una fuerte competencia y una innovación permanente de procesos, productos y servicios, con una premisa de máxima eficiencia y mínimo costo, dentro de parámetros de estándares de calidad y clase mundial. Este escenario implica que las empresas y organizaciones estén buscando permanentemente nuevas técnicas, herramientas, métodos, esquemas, metodologías y desarrollos que permitan mantener su mercado y en lo posible ampliarlo para su crecimiento, tal como lo establece (Alonso, 2006), se está en una sociedad que se caracteriza por la transición de la gestión de personas, la flexibilidad, la incertidumbre por los mercados desregulados, la formación de una economía interconectada en tiempo real, lo que incide en que las empresas y organizaciones, estén sujetas a cambios que ocasionan la rivalidad de la competencia, la aparición de nuevos empresarios, productos y servicios con un menor ciclo de vida, el uso de las tecnologías de información y comunicaciones, el desarrollo de la telefonía y computación móvil, los servicios orientados a clientes, la mercadotecnia y la innovación, entre otros.

En este marco, hay acuerdo sobre la importancia de la información en la gestión de la organización y de la tecnología avanzada en sistemas y aplicaciones de negocio que permiten a las empresas decidir (tomar decisiones) con más conocimientos, y establecer una alta eficiencia, en un mundo altamente competitivo y cada vez más incierto, donde la calidad y la oportunidad de la vigilancia tecnológica (VT) de la organización puede significar no sólo la diferencia entre pérdidas y ganancias, sino también la diferencia entre la supervivencia y la quiebra, (Bahrami, et al., 2012) además, uno de los factores determinantes para el éxito de la innovación en una organización es una adecuada vigilancia (Machin, et al., 2011), (Jespersen, et al., 2007), (Lievrouw, 2012) y (Kim et al., 2011).

Conceptos que también se adaptan y pueden ser aplicables a la educación pública universitaria, como en este caso la Universidad Nacional de Colombia sede Manizales, que no cuenta con un desarrollo de VT y que luego del análisis que se presenta en este artículo, se espera que a los diferentes grupos de interés se les esté informando todo lo relacionado con su área de conocimiento, en concordancia con los diversos desarrollos investigativos y académicos en diversos campos de la Universidad. La VT constituye un elemento de importante valor para cualquier organización, porque la observación y el análisis del entorno científico y tecnológico son herramientas de vital importancia para la toma de decisiones estratégicas que generen ventajas competitivas frente a otras organizaciones, además de considerarse como uno de los factores determinantes para lograr innovaciones en las organizaciones, puesto que se explica como el esfuerzo sistemático realizado por una organización para la búsqueda, análisis y difusión de información científica y tecnológica (Delgado, 2014).

La VT según (Palop y Vicente, 1999), (Sánchez y Palop, 2002) y (Martínez, 2007), se puede entender como un sistema organizado de observación y análisis del entorno, tratamiento y circulación interna de los hechos observados y posterior utilización en la empresa y permite detectar tendencias y tecnologías claves, captar y analizar acciones de los competidores y analizar los últimos desarrollos de los proveedores (Porter y Cunningham, 2005). (AENOR, 2006), Define VT según la norma UNE 166006 como un proceso organizado, selectivo y sistemático, para captar información del exterior y de la propia organización sobre ciencia y tecnología, seleccionarla, analizarla, difundirla y comunicarla, para convertirla en conocimiento con el fin de tomar decisiones con menor riesgo y poder anticiparse a los cambios.

Por otro lado, (Delgado y Arrebato, 2011), establecen que la VT es un conjunto de acciones coordinadas de búsqueda, tratamiento (filtrado, clasificación, análisis) y distribución de la información obtenida de modo legal, útil para distintas personas de una organización en un proceso de toma de decisiones y para la reflexión estratégica. Igualmente, a partir del modelo de las cinco (5) fuerzas de Porter (Porter, 1979), define cuatro tipos de vigilancia: Competitiva, comercial, del entorno y competitiva. De acuerdo con lo anterior (Izarral, et al., 2014), definen los tipos de vigilancia tal como sigue:

Vigilancia competitiva: Implica el análisis y seguimiento de los competidores actuales y potenciales. El destino de sus inversiones, sus productos, circuitos de distribución, tiempos de respuesta, tipo de clientes y grado de satisfacción, su organización, su capacidad financiera, la cadena de valor del sector, la situación de la empresa y su fuerza en dicha cadena de valor. La idea es buscar los competidores actuales y potenciales.

Vigilancia comercial: Abarca elementos referidos a mercados, los clientes, la evolución de sus necesidades, su solvencia, entre otros; los proveedores, la estrategia de lanzamiento de nuevos productos; la mano de obra en el sector y en la cadena de valor. Se encargan de analizar los datos referentes a productosmercados de clientes y proveedores (estudios de mercado, nuevos mercados, evolución de las necesidades de los clientes, solvencia de los clientes y nuevos productos ofrecidos por los proveedores). 
Vigilancia Tecnológica VT: Contempla los avances científicos y técnicos, fruto de la investigación básica y aplicada, los productos y servicios, los procesos de fabricación, los materiales, su cadena de transformación, las tecnologías y sistemas de información. Se encargan de analizar las tecnologías disponibles o que acaban de aparecer y capaces de intervenir en nuevos productos o procesos (patentes, proyectos investigación y desarrollo). Constituyen la vigilancia tecnológica propiamente dicha.

Vigilancia del entorno: Involucra la legislación y normativa, las barreras no arancelarias, entre otros elementos; el medioambiente y la evolución de su cuidado; la cultura, política, sociología que hay detrás de toda decisión de las personas. Contempla la detección de aquellos hechos exteriores que pueden condicionar el futuro, en áreas como la sociología, la política, el medio ambiente y las reglamentaciones e involucra aspectos relacionados con asociaciones empresariales, socios y observación in situ.

Además, es notable la necesidad de aplicar herramientas como la VT en las organizaciones, pues al realizarla estas sabrán qué está ocurriendo con el mercado y sus principales competidores, cuáles son los últimos avances tecnológicos que pueden ser aplicables a sus procesos, y qué investigaciones deben organizar que no se estén realizando en el mercado. Esto con el fin de identificar oportunidades y amenazas, tomar decisiones estratégicas y lograr mejorar la competitividad en las entidades que la apliquen (Arango et al., 2012).

Igualmente, la VT es un proceso de carácter informativo /documental selectivo que recopila y organiza información y documentos sobre un área de especialización muy concreta y que está dirigido específicamente a un grupo de usuarios o a varios conjuntos de usuarios, cuyos intereses están relacionados pero son diferentes. Por ello puede afirmarse que se trata de una moción que tiene cierta relación con una de las actividades clásicas de centros de documentación y bibliotecas, la difusión o diseminación selectiva de información, sistema de difusión a la carta que le ofrece a cada usuario las referencias de documentos correspondientes a sus temas de interés seleccionados a partir de todos los documentos o referencias recibidos durante un determinado período (Fernández, et al., 2009), en este sentido proponen el siguiente método, ver Figura 1:

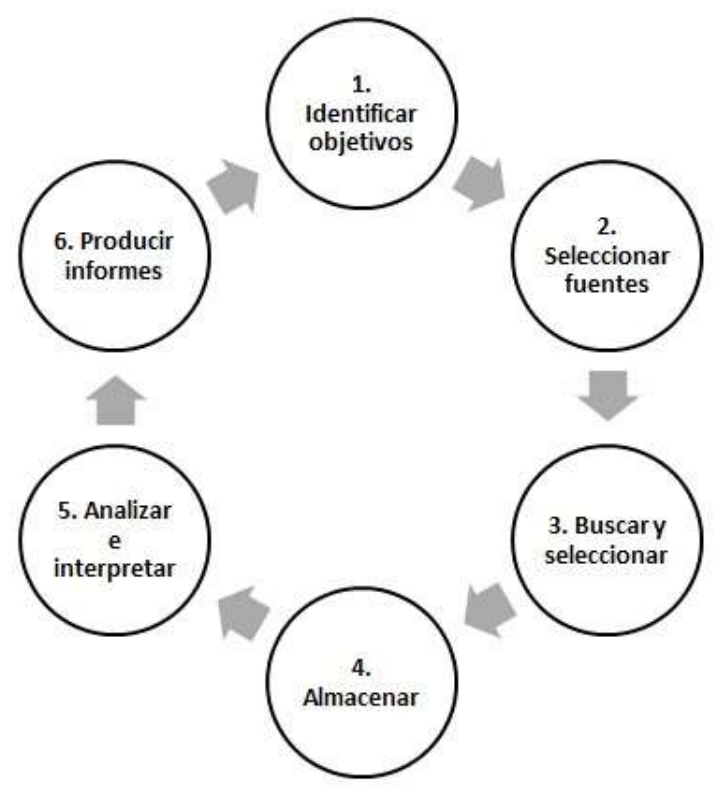

Fig. 1: Método de desarrollo de la VT. Adaptado de (Fernández, et al., 2009)

i) Identificación de objetivos: El primer paso que debe darse para establecer un sistema de VT es determinar las áreas temáticas, el punto de vista que interesa, la cobertura espacial y temporal, y las áreas idiomáticas o los tipos documentales que se van a vigilar.

ii) Selección de fuentes de información: En función de los objetivos se deben determinar las fuentes de información que se utilizarán para extraer la información relevante para cada entorno o ámbito de actuación.

iii) Buscar y seleccionar las fuentes de información: Se determinan los procesos adecuados para realizar búsquedas en las fuentes seleccionadas y se describirán y harán las recomendaciones oportunas sobre las herramientas de búsqueda, seguimiento y captación de información electrónica. 
iv) Almacenar la información: Es importante conocer las herramientas adecuadas que permitan el almacenamiento de la información, así como su gestión y puesta a disposición de los usuarios que forman parte del proceso de vigilancia.

v) Analizar e interpretar la información: La VT es un proceso científico interpretativo e incluye el análisis de la información recopilada con el fin de detectar tendencias, novedades y avances.

vi) Producir informes de VT: Los informes de VT son herramientas de trabajo al servicio de la toma de decisiones y por ello debe establecerse la periodicidad, los criterios e índice del contenido y la estructura de presentación de los datos.

Pero para el logro de lo mostrado en el modelo anterior, es necesario según (Zhua y Porter, 2002), considerar una serie de factores críticos, incluyendo: i) Participación de los usuarios en la formulación y el proceso analítico; ii) La necesidad de representaciones de las principales conclusiones, considerando la naturaleza del cambio tecnológico; iii) Centrarse justo en lo que el usuario prefiere (mezcla adecuada de aspectos relacionados con la tecnología y el contexto); iv) Credibilidad (del analista, los métodos utilizados y los datos empleados); y v) Comunicaciones (derecho de información en forma clara y accesible).

Para el caso de una universidad (Delgado, et al., 2010), establecen que la VT es muy importante para el éxito de los procesos de I+D+i (investigación, desarrollo e innovación). Se define como el proceso organizado, selectivo y permanente, de captar información del exterior y de la propia organización sobre ciencia y tecnología, seleccionarla, analizarla, difundirla y comunicarla, para convertirla en conocimiento para tomar decisiones con menor riesgo y poder anticiparse a los cambios. La identificación adecuada de la fuente de información es vital para la vigilancia tecnológica, siendo cada vez más usadas las páginas web, en las que están las bases de datos de patentes, revistas científicas, portales de universidades. El análisis de esa información permite tomar mejores decisiones y anticiparse a los cambios sistemáticos del entorno.

En las universidades y centros de investigación se requiere implementar una metodología de vigilancia tecnológica que involucre la mayor cantidad de actores posibles y el uso de herramientas de captura, análisis, procesamiento y difusión de la información, así como de indicadores de control de este proceso y de los propios programas estratégicos de investigación. Para este caso se recomienda el uso de los procesos definidos en la norma UNE 166006 EX Gestión de la I+D+i (AENOR, 2006): Sistema de Vigilancia Tecnológica.

Igualmente, considerando las principales necesidades de información de la Universidad Nacional de Colombia sede Manizales, se han identificado fuentes de información y recursos disponibles tales como: i) Documentación propia; ii) Personal docente y estudiantil; iii) Personas o entidades externas de interés; iv) Fuentes documentales del sistema de bibliotecas como: libros de texto, revistas, catálogos, bases de datos de revistas y de tesis de pre y posgrado, así como libros de autoría de profesores de la universidad; v) Recursos de información en Internet: portales, páginas especializadas, blogs; vi) Registro de asistencia a congresos, seminarios, ferias y exposiciones de parte de los docentes; y vii) Publicaciones de los docentes.

Y desde la diversidad de fuentes a considerar se cuenta con información científico-tecnológica, información relativa a noticias, eventos, cursos de educación continuada, oferta y demanda tecnológica, proyectos de investigación y convocatorias, entre otras.

\section{MATERIALES Y MÉTODOS}

Dadas las condiciones y las características de la información a obtener, fue necesario emplear un método de investigación cualitativa, el cual según (Pérez 1994) y (Marulanda, et al., 2013), busca comprender por medio de significados y desde una perspectiva holística, el conjunto de cualidades interrelacionadas que caracterizan a un determinado fenómeno. Además de ser una investigación exploratoria, que permite analizar e identificar las principales variables del modelo de evaluación, una investigación descriptiva, que permite conocer las situaciones predominantes con la representación exacta de los procesos, y una investigación de tipo correlacional que permite encontrar la relación entre las variables. El tratamiento estadístico implico un análisis de correlación y un análisis de componentes principales.

\section{Determinación de la muestra}

Se aplicó la encuesta de manera personal a una muestra de la población de 300 estudiantes de diversos programas de pregrado de la sede palogrande de la Universidad Nacional sede Manizales. Previamente a la valoración, se desarrolló una prueba piloto de la encuesta con expertos sobre el tema, además de una revisión con algunos de los actores académicos. Para la estructuración del cuestionario se formularon 
preguntas en escala Likert, las cuales se calificaron de 1 a 5 , donde 1 , se está en desacuerdo o no realizado, 2, realizado parcialmente, 3 , realizado en intervalos, 4 , realizado con regularidad y 5 realizado completamente

\section{Variables evaluadas}

Las variables se fundamentan en los planteamientos desarrollados por (Bernete, 2009) y (Arango, et al., 2012), de los cuales se hizo un análisis de taxonomía, considerando las interrelaciones y las perspectivas dinámicas de la educación y la VT, por medio de métricas, herramientas e indicadores específicos, ligados a las consideraciones individuales. Estas se agruparon tal como se observa en la Tabla 1. Para revisión de los resultados de la encuesta, revisar en https://drive.google.com/file/d/0BzMqd65Rck5Z20xbk1kcTZyWDg/view?usp=sharing.

Tabla 1: Variables evaluadas

\begin{tabular}{|l|l|}
\hline \multicolumn{1}{|c|}{ Categorías } & \multicolumn{1}{c|}{ Variables } \\
\hline Dirección & Directivos, interés y bases de datos. \\
\hline $\begin{array}{l}\text { Medios de } \\
\text { Comunicación }\end{array}$ & $\begin{array}{l}\text { Foros, unimedios, bases de datos, libros de investigación, portal } \\
\text { institucional, mensajes de alerta, revistas y sistema de biblioteca. }\end{array}$ \\
\hline VT individual & $\begin{array}{l}\text { Otras fuentes, opinión, organización, proyectos, eventos, } \\
\text { disponibilidad, uso de móvil y web, redes sociales y móvil e interés. }\end{array}$ \\
\hline
\end{tabular}

\section{RESULTADOS Y DISCUSIÓN}

El proceso de recolección de datos se hizo personalmente con los estudiantes de diferentes semestres de programas de pregrado de ingeniería civil, ingeniería industrial, ingeniería química, ingeniería electrónica, ingeniería física, ingeniería eléctrica, matemáticas, gestión cultural y administración de empresas diurna y nocturna, además de hacer un muestreo aleatorio de comprobación. Se presenta a su vez la matriz de correlación, ver Tabla 2, la cual indica un alto grado de correlación entre las variables evaluadas, lo que hace posible aplicar el método de componentes principales. En la categoría dirección institucional para la VT, los resultados se graficaron tal como se observa en la Figura 2.

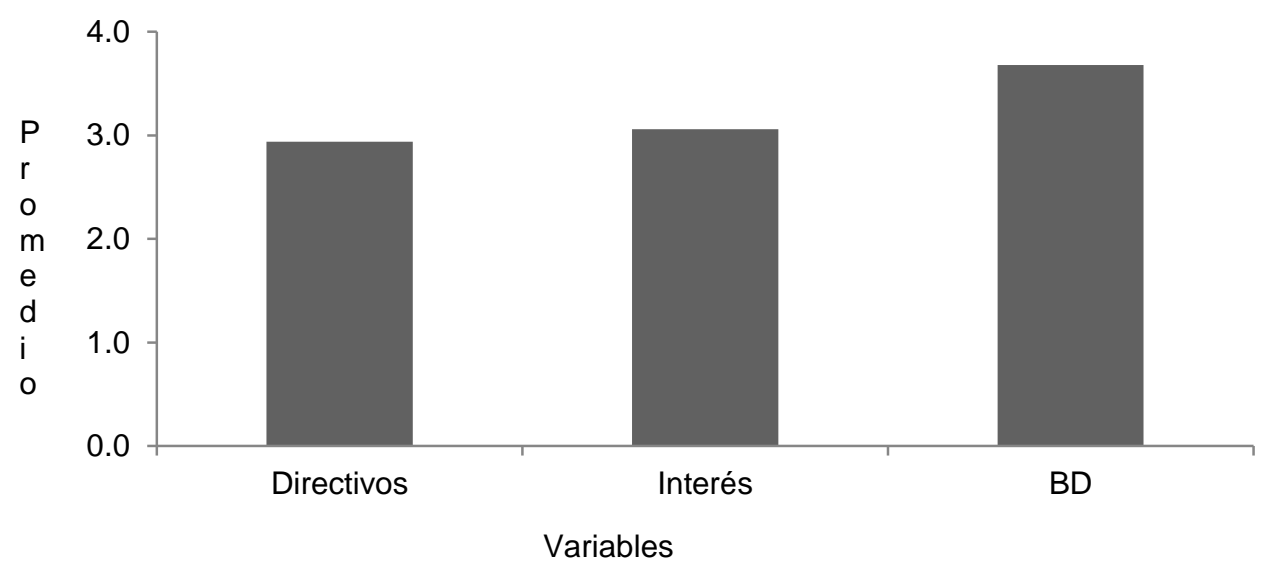

Fig. 2: Dirección institucional de la VT

De acuerdo con la escala de calificación, el promedio de las variables evaluadas está alrededor de 3.2, lo que muestra una calificación baja en la gestión estratégica institucional para el desarrollo de la VT en la Universidad Nacional de Colombia sede Manizales. Se destacan valores por debajo del promedio frente a la percepción que las directivas de la Universidad no reconocen adecuadamente las ventajas de la VT y tiene un bajo compromiso para apoyarla, lo que muestra pocos esfuerzos para gestionar la VT y hace necesario establecer estrategias para su mejoramiento. Mientras se cuenta con resultados por encima del promedio en bases de datos de artículos científicos que contienen información relevante en las áreas de interés de los estudiantes, producto manifiesto del direccionamiento de los líderes de la Universidad, para garantizar desde el presupuesto, acceso a diversas bases de datos electrónicas pare el beneficio del estamento estudiantil. 
Tabla 2: Matriz de correlación (valor p) entre variables

\begin{tabular}{|c|c|c|c|c|c|c|c|c|c|c|c|c|c|c|c|c|c|c|c|}
\hline \multirow{2}{*}{$\begin{array}{l}\text { Variables } \\
\text { Directivos }\end{array}$} & & & & & & & & & & & & & & & & & & & \\
\hline & & & & & & & & & & & & & & & & & & & \\
\hline Interés & 0,22 & & & & & & & & & & & & & & & & & & \\
\hline BDD & 0,20 & 0,15 & & & & & & & & & & & & & & & & & \\
\hline Foros & 0,25 & 0,25 & 0,29 & & & & & & & & & & & & & & & & \\
\hline Unimed & 0,19 & 0,25 & 0,18 & 0,38 & & & & & & & & & & & & & & & \\
\hline $\mathrm{BDc}$ & 0,17 & 0,20 & 0,39 & 0,30 & 0,26 & & & & & & & & & & & & & & \\
\hline Libros & 0,14 & 0,14 & 0,24 & 0,39 & 0,18 & 0,48 & & & & & & & & & & & & & \\
\hline Portal & 0,10 & 0,29 & 0,17 & 0,30 & 0,33 & 0,26 & 0,32 & & & & & & & & & & & & \\
\hline Alerta & 0,12 & 0,26 & 0,18 & 0,41 & 0,32 & 0,27 & 0,30 & 0,43 & & & & & & & & & & & \\
\hline Revist & 0,14 & 0,13 & 0,21 & 0,28 & 0,23 & 0,38 & 0,35 & 0,26 & 0,40 & & & & & & & & & & \\
\hline Biblio & 0,12 & 0,21 & 0,25 & 0,27 & 0,15 & 0,48 & 0,41 & 0,23 & 0,27 & 0,43 & & & & & & & & & \\
\hline Otrasf & 0,17 & 0,22 & 0,14 & 0,30 & 0,12 & 0,20 & 0,22 & 0,17 & 0,16 & 0,12 & 0,23 & & & & & & & & \\
\hline Opin & 0,21 & 0,15 & 0,17 & 0,24 & 0,18 & 0,23 & 0,17 & 0,18 & 0,20 & 0,16 & 0,14 & 0,37 & & & & & & & \\
\hline Organ & 0,12 & 0,04 & 0,11 & 0,12 & 0,10 & 0,13 & 0,20 & 0,11 & 0,05 & 0,12 & 0,10 & 0,11 & 0,14 & & & & & & \\
\hline Proy & 0,15 & 0,05 & 0,16 & 0,20 & 0,13 & 0,14 & 0,09 & 0,02 & 0,04 & 0,15 & 0,08 & 0,16 & 0,24 & 0,49 & & & & & \\
\hline Event & 0,17 & 0,26 & 0,17 & 0,29 & 0,26 & 0,20 & 0,17 & 0,19 & 0,30 & 0,20 & 0,21 & 0,15 & 0,17 & 0,10 & 0,16 & & & & \\
\hline Dispon & 0,15 & 0,11 & 0,17 & 0,20 & 0,20 & 0,22 & 0,26 & 0,18 & 0,22 & 0,18 & 0,17 & 0,12 & 0,21 & 0,16 & 0,18 & 0,49 & & & \\
\hline móvilyweb & 0,14 & 0,22 & 0,17 & 0,21 & 0,21 & 0,20 & 0,20 & 0,19 & 0,20 & 0,12 & 0,14 & 0,19 & 0,24 & 0,23 & 0,17 & 0,18 & 0,20 & & \\
\hline Redeso & 0,07 & 0,05 & 0,14 & 0,11 & 0,17 & 0,11 & 0,05 & 0,16 & 0,11 & 0,11 & 0,04 & 0,08 & 0,13 & 0,13 & 0,18 & 0,08 & 0,05 & 0,56 & \\
\hline móvilyar & 0,11 & 0,06 & 0,12 & 0,12 & 0,07 & 0,16 & 0,02 & 0,05 & 0,00 & 0,04 & 0,14 & 0,11 & 0,05 & 0,23 & 0,16 & 0,08 & 0,08 & 0,24 & 0,32 \\
\hline
\end{tabular}

En este sentido el desarrollar un observatorio tecnológico permitiría gestionar el conocimiento a través de la vigilancia del entorno científico y tecnológico, para generar nuevos conocimientos. Además de establecer vínculos con otras universidades para compartir y recibir información (Muñoz, et al., 2006).

Desde la categoría uso de medios de comunicación para la VT, los resultados se graficaron tal como se observa en la Figura 3.

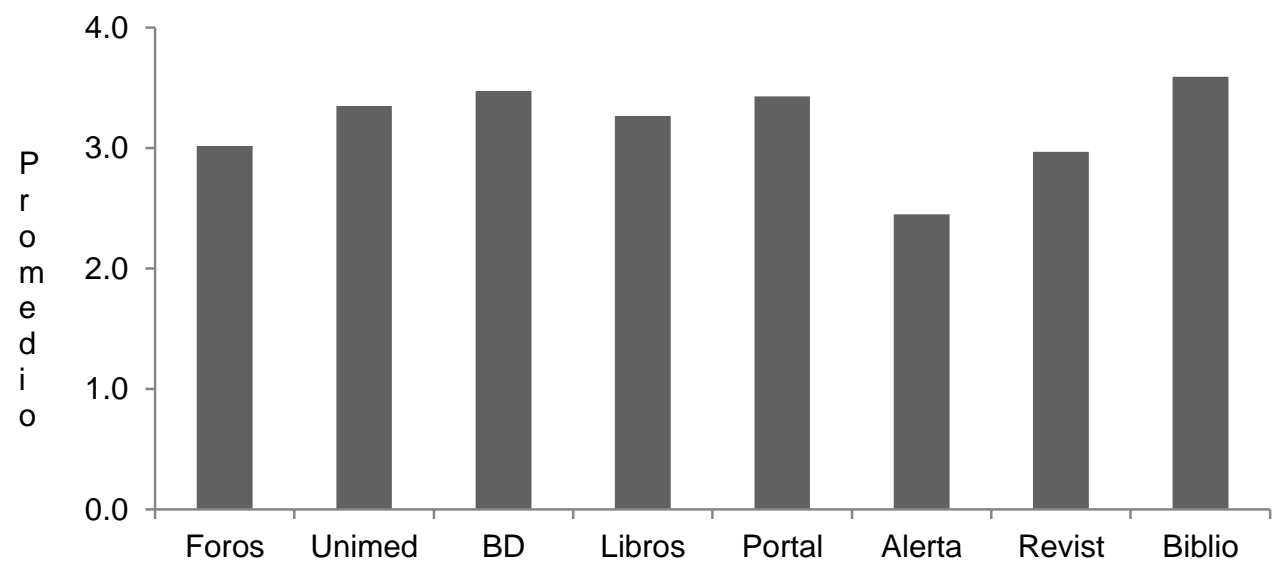

Variables

Fig. 3: Medios de comunicación para la VT 
De acuerdo a la escala de calificación, el promedio de las variables evaluadas está alrededor de 3.2, lo que muestra una calificación baja en la comunicación para el desarrollo de la VT en la Universidad Nacional de Colombia sede Manizales. Se destacan valores por debajo del promedio frente a la falta de visibilidad de los resultados de los foros institucionales, además de las publicaciones en las revistas por parte de los docentes universitarios y los resultados de investigaciones en revistas propias, igualmente hay poca difusión de medios de alerta tecnológica que permita a los grupos de interés tener conocimiento preciso y detallado de los avances en sus áreas de trabajo, lo que hace necesario plantear estrategias desde las aulas de clase para una mayor uso de estas herramientas. Además de lo planteado por (González, et al., 2013), frente a la necesidad de contar con un sistema de gestión de la información a nivel de la universidad, que permita realizar análisis para la toma de decisiones referidas a los procesos universitarios.

Mientras se cuenta con resultados por encima del promedio en los recursos bibliográficos de la biblioteca, bases de datos de artículos científicos que contienen información relevante en las áreas de interés de los estudiantes, portal institucional y el medio impreso Unimedios, el cual entrega información de resultados de investigación, lo que muestra el interés institucional, para entregar herramientas de trabajo a la comunidad académica y también divulgar los resultados de los trabajos investigación y de proyección universitaria. Desde la categoría uso personal de la VT, los resultados se graficaron tal como se observa en la Fig. 4.

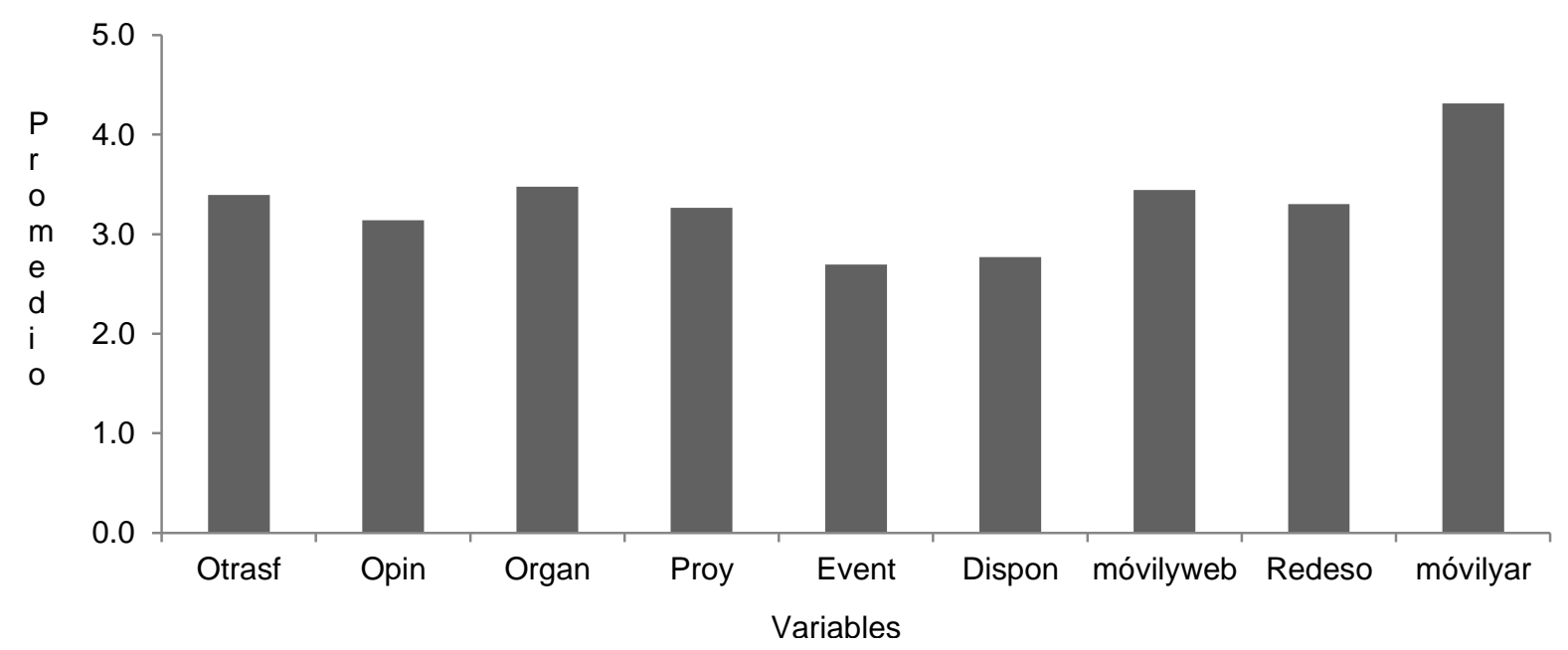

Fig. 4: Uso personal de la VT

De acuerdo a la escala de calificación, el promedio de las variables evaluadas está alrededor de 3.3, lo que muestra una calificación baja en el uso personal de la VT por parte de los estudiantes de la Universidad Nacional de Colombia sede Manizales. Se destacan valores por debajo del promedio frente a disponer información de eventos, disponer de información de producción científica de la Universidad y enriquecer la información de sus clases incluyendo la opinión de expertos, lo que muestra que a pesar de contar con algunas herramientas institucionales para desarrollar actividades académicas, los estudiantes limitan su quehacer, incluso para pedir apoyo a expertos, como podría ser el caso de los profesores.

Mientras se cuenta con resultados por encima del promedio en el interés de utilizar su teléfono móvil inteligente para estar enterado de todo lo relacionado con sus áreas educativas de interés, en menor medida utilizan su teléfono inteligente para revisar periódicamente determinados sitios web que ofrecen información de su área educativa de interés y organizar y archivar la información para su posterior recuperación. Considerando la gran cantidad de variables utilizadas para medir la vigilancia tecnológica para estudiantes, es necesario aplicar una técnica estadística de componentes principales que permita realizar una síntesis de la información, reflejada en la reducción de la dimensiones (número de variables) procurando la menor perdida de información posible (ver tabla 3).

Sobre la base de estos resultados (leer porcentaje acumulado de las líneas 1, 2, 3, 4, 5 y 6) se establece lo siguiente: el primer componente explica el $24 \%$ de los resultados, el segundo explica el $33 \%$, el tercero explica el $40 \%$, el cuarto explica el $46 \%$, el quinto explica el $52 \%$ y el sexto explica el $57 \%$. Se procede entonces a asignar a cada variable evaluada un componente, de acuerdo con el número mayor de los seis componentes, como se observa en la Tabla 4 y el resultado de dicha asignación se encuentra en la Tabla 5. 
Tabla 3: Componentes principales de las variables

\begin{tabular}{|l|r|r|r|r|r|r|}
\hline \multirow{2}{*}{ Componente } & \multicolumn{4}{|c|}{ Autovalores iniciales } & \multicolumn{2}{c|}{ Sumas de las saturaciones al cuadrado de la extracción } \\
\cline { 2 - 7 } & Total & $\begin{array}{c}\text { \% de la } \\
\text { varianza }\end{array}$ & $\begin{array}{c}\text { \% } \\
\text { acumulado }\end{array}$ & \multicolumn{1}{c|}{ Total } & $\begin{array}{c}\text { \% da } \\
\text { varianza }\end{array}$ & \% acumulado \\
\hline 1 & 4,779 & 23,897 & 23,897 & 4,779 & 23,897 & 23,89 \\
\hline 2 & 1,781 & 8,903 & 32,800 & 1,781 & 8,903 & 32,80 \\
\hline 3 & 1,331 & 6,656 & 39,455 & 1,331 & 6,656 & 39,45 \\
\hline 4 & 1,284 & 6,419 & 45,874 & 1,284 & 6,419 & 45,87 \\
\hline 5 & 1,144 & 5,719 & 51,594 & 1,144 & 5,719 & 51,59 \\
\hline 6 & 1,008 & 5,040 & 56,634 & 1,008 & 5,040 & 56,63 \\
\hline
\end{tabular}

Tabla 4: Matriz de componentes de las variables para asignación

\begin{tabular}{|l|c|c|c|c|c|c|}
\hline & \multicolumn{7}{|c|}{ Componente } \\
\cline { 2 - 7 } & 1 & 2 & 3 & 4 & 5 & 6 \\
\hline Directivos &, 382 &, 101 &, 044 &, 281 &, 284 &, 313 \\
\hline Interés &, 439 &,- 126 &, 360 &, 111 &, 221 &, 137 \\
\hline BDD &, 483 &, 000 &,- 187 &,- 093 &, 078 &, 348 \\
\hline Foros &, 642 &,- 117 &, 091 &, 087 &, 112 &,- 097 \\
\hline Unimed &, 322 &,- 063 &, 431 &, 044 &,- 126 &,- 145 \\
\hline BDc &, 633 &,- 170 &,- 328 &,- 245 &, 043 &, 195 \\
\hline Libros &, 511 &,- 247 &, 513 &,- 153 &,- 008 &,- 097 \\
\hline Portal &, 535 &,- 216 &, 291 &,- 166 &,- 042 &,- 326 \\
\hline Alerta &, 582 &,- 319 &, 264 &,- 058 &,- 138 &,- 268 \\
\hline Revist &, 519 &,- 264 &,- 243 &, 533 &,- 147 &,- 159 \\
\hline Biblio &, 525 &,- 278 &,- 356 &, 548 &, 557 &, 163 \\
\hline Otrasf &, 440 &, 066 &,- 015 &, 214 &, 579 &,- 050 \\
\hline Opin &, 465 &, 154 &, 041 &, 297 &, 398 &,- 139 \\
\hline Organ &, 339 &, 499 &,- 387 &, 149 &,- 164 &,- 351 \\
\hline Proy &, 355 &, 517 &,- 340 &, 320 &,- 087 &,- 280 \\
\hline Event &, 503 &,- 059 &, 195 &, 404 &,- 416 &, 287 \\
\hline Dispon &, 473 &, 017 &, 003 &, 400 &,- 476 &, 539 \\
\hline móvilyweb &, 405 &, 475 &, 307 &,- 278 &,- 005 &, 431 \\
\hline Redeso &, 326 &, 567 &, 316 &,- 464 &,- 072 &, 007 \\
\hline móvilyar &, 259 &, 507 &,- 054 &,- 287 &,- 030 &, 315 \\
\hline
\end{tabular}

Tabla 5. Asignación de variables a componentes

\begin{tabular}{|l|l|}
\hline Variable & Representada por el factor \\
\hline Directivos & 1: Vigilancia institucional \\
\hline Interés & 1: Vigilancia institucional \\
\hline BDD & 1: Vigilancia institucional \\
\hline Foros & 1: Vigilancia institucional \\
\hline Unimed & 3: Periódico y libros \\
\hline BDc & 1: Vigilancia institucional \\
\hline Libros & 3: Periódico y libros \\
\hline Portal & 1: Vigilancia institucional \\
\hline Alerta & 1: Vigilancia institucional \\
\hline Revist & $4:$ Revistas y biblioteca \\
\hline Biblio & 4: Revistas y biblioteca \\
\hline Otrasf & 5: Otras fuentes \\
\hline Opin & 1: Vigilancia institucional \\
\hline Organ & 2: Móvil para estudiantes \\
\hline
\end{tabular}


Tabla 5: continuación

\begin{tabular}{|l|l|}
\hline Proy & 2: Móvil para estudiantes \\
\hline Event & 1: Vigilancia institucional \\
\hline Dispon & 6: Uso de web \\
\hline móvilyweb & 6: Uso de web \\
\hline Redeso & 2: Móvil para estudiantes \\
\hline móvilyar & 2: Móvil para estudiantes \\
\hline
\end{tabular}

Con estos factores se hizo la matriz de correlaciones para corroborar que efectivamente los componentes están bien agrupados. Lo resultados mostraron un nivel alto de significancia entre los nuevos 6 factores y ratifica que el la vigilancia tecnológica para estudiantes de pregrado de la Universidad Nacional de Colombia sede Manizales, se está generando desde la vigilancia institucional, móvil para estudiantes, periódico y libros, revistas y biblioteca, otras fuentes y uso de la web.

\section{CONCLUSIONES}

Dadas las dinámicas de cambio del mundo actual y considerando la universidad como un eje fundamental del desarrollo social, es necesario que cuente con herramientas que permitan avanzar en procesos de gestión del conocimiento para la comunidad académica y empresarial, desde la vigilancia tectológica, más allá de contar con diversos medios de comunicación, publicidad y promoción, integrando además la información que se tiene.

Se reconocen los esfuerzos tan importantes que viene haciendo a Universidad, desde un presupuesto público exiguo, para mantener su portal, sus bases de datos, sus revistas, sus medio de difusión y en este sentido, lo que se está haciendo en cuanto a VT, aporta al mejoramiento de los procesos de desarrollo, pero indudablemente se percibe de parte de los estudiantes la necesidad de avanzar más en este importante tema, dada su importancia y las diversas posibilidades que tienes ellos con el acceso y uso de diversos medios y herramientas tecnológicas.

Los resultados de ésta investigación y las demás, relacionadas con el desarrollo e innovación desde la universidad, debe considerar a sus grupos de interés, dada la necesidad de aplicar el conocimiento a las circunstancias de las diversas comunidades, desde una mirada de bien social en el marco del desarrollo económico, político y social y es por medio de este tipo herramientas que esto se puede lograr. Para este caso es fundamental considerar en espacios de desarrollo de vigilancia tecnológica a los estudiantes.

Si bien la universidad debe propender por la VT, es también importante que los estudiantes aprovechen al máximo los recursos y las herramientas con las que cuentan y en este sentido, según el estudio los niveles de aprovechamiento es baja, lo que implicaría plantear estrategias que permitan apropiar aún más el concepto de VT en la comunidad académica.

\section{AGRADECIMIENTOS}

Este trabajo es parte de los resultados del proyecto de investigación titulado "Desarrollo de una aplicación móvil para alertas tecnológicas", que fue registrado y financiado por la Dirección de Investigación de la Universidad Nacional de Colombia sede Manizales, con código Hermes 23058.

\section{REFERENCIAS}

AENOR. Norma Española Experimental UNE 166006 Gestión de la I+D+i: Sistema de Vigilancia Tecnológica. AENOR. (2006)

Alonso, L. F. El imaginario Managerial. Política y sociedad, 127-151. (2006)

Arango, B., Tamayo, L., y Fadul, A., Vigilancia tecnológica: metodologías y aplicaicones. GPT Gestión de las Personas y Tecnología, 1-4. (2012)

Bahrami, M., Arabzad, M., y Ghorbani, M., Innovation In Market Management By Utilizing Business Intelligence: Introducing Proposed Framework. Procedia - Social and Behavioral Sciences, 160-167 (2012)

Bernete, F., Usos de las TIC, Relaciones sociales y cambios en la socialización de las y los jovenes. CIES PAL/CHAS QUI , Revista Latinoamericana de Comunicación, 97-114 (2009) 
Delgado, M. I. Vigilancia tecnológica como factor clave para el éxito en la I+D+i: aplicación en el ámbito universitario. (2014). http://www.delfo. Acceso: 1 de Diciembre de 2014)

Delgado, M., y Arrebato, L., Diagnóstico integrado de la vigilancia tecnológica en organizaciones. Ingeniería Industrial, 1-6. (2011)

Delgado, M., Infante, M., Abreu, Y., García, B., Infante, O., y Díaz, A., Metodología de vigilancia tecnológica en universidades y centros de investigación. Revista CENIC. Ciencias Biológicas, 1-13. (2010)

Fernández, B., Pérez, S., y Del Valle, F. Metodología para la implantación de sistemas de vigilancia tecnológica y documental: El caso del proyecto INREDIS. Investigación bilbiotecológica, 149-177. (2009)

González, K., Sánchez-Morles, J. G., y Caira-Tovar, N. M., Herramientas Informativas para la Vigilancia Tecnológica en Diseños Curriculares de Universidades Públicas (Information Tools for Technological Monitoring on State University Curriculum Design). GECONTEC: Revista Internacional de Gestión del Conocimiento y la Tecnología, 1(2) (2013)

Izarral, A., Sánchez, J., y Caira, T. Ejes de Vigilancia Tecnológica Aplicados en Universidades con estudios a distancia. GECONTEC: Revista Internacional de Gestión del Conocimiento y la Tecnología, 1-10. (2014).

Jespersen, J. L., Albrechtslund, A., Øhrstrøm, P., Hasle, P., y Albretsen, J., Surveillance, persuasion, and panopticon. In Persuasive technology (pp. 109-120). Springer Berlin Heidelberg. (2007)

Kim, A. R., Rhee, S. Y., Seok, S. J., Lee, H. C., y Kim, Y. B., A Surveillance System for a Farm. In Informatics Engineering and Information Science (pp. 273-284). Springer Berlin Heidelberg. (2011)

Lievrouw, L. A., The Next Decade in Internet Time: Ways ahead for new media studies. Information, Communication \& Society, 15(5), 616-638. (2012)

Machin, L., Raytor, M., y Bello, L., Propuesta de proceso de vigilancia tecnológica para el observatorio de tecnología educativa. Edutec- e, 1-14 (2011)

Martínez, L., La información como herramienta de la vigilancia estratégica. Revista beroamericana sobre usuarios de información, 1-10. (2007)

Marulanda, C., López, M., y Castaño, J., Servicios en la nube para secretarías de vivienda. Revista virtual Universidad Católica del Norte, 111-121. (2013)

Muñoz, J., Marín, M., y Vallejo, J., La vigilancia tecnológica en la gestión de proyectos de I+ D+ i: recursos y herramientas. El profesional de la información, 15(5), 411-419. (2006)

Palop, F., y Vicente, J. M., Vigilancia tecnológica e inteligencia competitiva: su potencial para la empresa española. . Madrid: Cotec. (1999)

Porter, A., y Cunningham, S., Tech mining. Exploiting New Technologies for Competitive Advantage, . New Jersey: Wiley-Interscience. (2005)

Porter, M. E. How Competitive Forces Shape Strategy. Harvard Business Review, 57(1), 137-145. (1979)

Sánchez, J., y Palop, F., Herramientas de Software para la práctica de la Inteligencia Competitiva en la empresa. . Valencia: Triz XXI. (2002)

Zhua, D., y Porter, A. Automated extraction and visualization of information fortechnological intelligence and forecasting. Technological Forecasting \& Social Change, 495-506. (2002)

White, J.K., Phase Equilibria in Hydrocarbon-Alcohol Systems, http:www.jpt.uk/41.6.htm, ISSN: 0888-5555, J. Pet. Technology 41(6), 37-45 (2013) 
